\title{
Apolipoprotein C-III Strongly Correlates with Activated Factor VII-Anti-Thrombin Complex: An Additional Link between Plasma Lipids and Coagulation
}

\author{
Nicola Martinelli ${ }^{1}$ Marcello Baroni ${ }^{2}$ Annalisa Castagna ${ }^{1}$ Barbara Lunghi ${ }^{2}$ Filippo Stefanoni ${ }^{1}$ \\ Federica Tosi ${ }^{1}$ Jacopo Croce ${ }^{1}$ Silvia Udali ${ }^{1}$ Barry Woodhams ${ }^{3}$ Domenico Girelli ${ }^{1}$ \\ Francesco Bernardi ${ }^{2}$ Oliviero Olivieri ${ }^{1}$
}

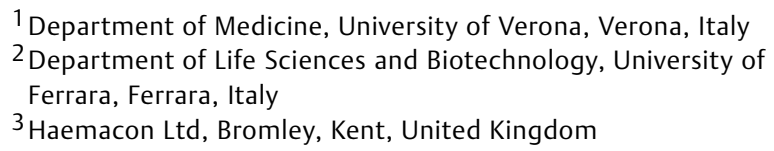

Address for correspondence Nicola Martinelli, MD, PhD, Department of Medicine, University of Verona, Verona, Italy

(e-mail: nicola.martinelli@univr.it).

Thromb Haemost 2019;119:192-202.

\begin{abstract}
Keywords

- activated factor VII-anti-thrombin complex

- APOC3 gene polymorphism

- apolipoprotein C-III

- coagulation

- plasma lipids

Activated factor VII-anti-thrombin (FVlla-AT) complex is a potential biomarker of prothrombotic diathesis reflecting FVIla-tissue factor (TF) interaction and has been associated with mortality in patients with coronary artery disease (CAD). Previous data indicated plasma lipids as predictors of FVIla-AT variability, and plasma lipoproteins as potential stimulators of the coagulation cascade. Our aim was to evaluate the relationships between FVIla-AT plasma concentration and a broad apolipoprotein profile (including ApoA-I, ApoB, ApoC-III and ApoE). Within the framework of the observational Verona Heart Study, we selected 666 subjects ( 131 CAD-free and 535 CAD, 75.4\% males, mean age: $61.1 \pm 10.9$ years) not taking anticoagulant drugs and for whom plasma samples were available for both FVIla-AT assay and a complete lipid profile. Plasma concentration of FVIla-AT levels significantly and directly correlated with total and high-density lipoprotein cholesterol, triglycerides, ApoA-I, ApoC-III and ApoE levels. ApoC-III showed the strongest correlation $\left(R=0.235, p=7.7 \times 10^{-10}\right)$, confirmed in all the sub-group analyses (males/females and CAD/CAD-free). Only ApoC-III remained associated with FVIla-AT plasma concentration, even after adjustment for sex, age, CAD diagnosis, body mass index, renal function, smoking status, lipid-lowering therapies and FVIla levels. The APOC3 gene locus-tagging polymorphism rs964184, previously linked with cardiovascular risk and plasma lipids by genome-wide association studies, was associated with both ApoC-III and FVIlla-AT plasma concentration. Our results indicate a strong association between ApoC-III and FVIla-AT levels, thereby suggesting that an increased ApoC-III concentration may identify subjects with a prothrombotic diathesis characterized by an enhanced TF-FVlla interaction and activity.
\end{abstract}

\section{Introduction}

Plasma lipids play a crucial role in the pathogenesis of cardiovascular disease (CVD). ${ }^{1}$ Lipoproteins are the main actors of a finely tuned system to transport lipids through the whole body according to its metabolic needs. Lipopro- teins have a mono-layer phospholipid and cholesterol outer shell and heterogeneous lipid core. Apolipoproteins are embedded in the outer shell, both stabilizing the structure and determining their functional characteristics.

Alterations in levels of plasma lipids are well recognized among the most important risk factors of CVD, and lipid- received

July 21,2018

accepted after revision

November 6, 2018 (c) 2019 Georg Thieme Verlag KG Stuttgart · New York
DOI https://doi.org/

10.1055/s-0038-1676817. ISSN 0340-6245. 
lowering drugs are among the most widely used drugs in Western world. ${ }^{2,3}$ The increase of cardiovascular risk due to alterations in plasma lipids concentration is usually attributed to atherosclerosis-related processes. However, lipoproteins are endowed with various biological properties and have been also demonstrated to influence the coagulation pathway.

Coagulation needs lipids and the cascade is greatly accelerated by lipid binding. ${ }^{4}$ Several coagulation factors are equipped with specialized membrane phospholipid-binding domains, which are essential for proper clot formation. ${ }^{4}$ Tissue factor (TF), whose interaction with activated factor VII (FVIIa) starts the coagulation cascade, is not only an integral membrane protein but its ectodomain is also involved in phospholipid interactions which enhance FVIIa activity. ${ }^{5}$ Thrombin generation is dependent on the assembly of the pro-thrombinase complex on a phospholipid surface. ${ }^{6}$ In clinical studies, plasma levels of both cholesterol and triglycerides have been directly correlated with plasma levels of coagulation factors, especially with the vitamin K-dependent ones, ${ }^{7}$ as well as with thrombin generation. ${ }^{8}$ Experimental data suggest that all plasma lipoproteins stimulate coagulation cascade, with triglyceride-rich lipoproteins (TRLs) being apparently the most efficient. ${ }^{9,10}$

Activated FVII-anti-thrombin (FVIIa-AT) complex is an indirect marker of intravascular exposure of TF and, consequently, of activation of the extrinsic coagulation pathway. While still incompletely understood, the transition from inactive (encrypted) to active (decrypted) form of TF and its binding and activation of FVII is the key initiator of coagulation cascade. ${ }^{11-13}$ Both AT and TF pathway inhibitor (TFPI) acts as inhibitors of the TF-FVIIa pathway. TFPI forms with TF, FVIIa and activated coagulation FXa, a stable quaternary complex which remains bound to the cellular surface. On the other hand, once the TF-FVIIa-AT complex is formed, FVIIa loses affinity for TF and FVIIa-AT is released and accumulates in the plasma where it can be measured. ${ }^{14}$ Therefore, FVIIa-AT plasma levels indirectly reflect TF-FVIIa interaction and have been suggested as a biomarker of pro-thrombotic diathesis. ${ }^{15-17}$ A slight increase in FVIIa-AT plasma concentration was observed in subjects with previous arterial and/or venous thrombosis, ${ }^{15}$ as well as in survivors after myocardial infarction. ${ }^{16}$ In the setting of primary prevention of coronary artery disease (CAD) within the Stockholm study of 60-year-old individuals, FVIIa-AT had no predictive value, ${ }^{16}$ while in the secondary prevention setting within the Verona Heart Study (VHS) population, high FVIIa-AT levels predicted total and cardiovascular mortality in patients with clinically stable CAD. ${ }^{17}$ This suggests that FVIIa-AT levels may be more related with later thrombotic complications of CAD, rather than with the early development of atherosclerotic plaques. Accordingly, a recent analysis in participants to the large Cardiovascular Health Study has shown that high FVIIa-AT levels may reflect an increased risk of mortality. ${ }^{18}$

In our previous study, high-density lipoprotein (HDL) cholesterol and triglycerides were independent predictors of FVIIa-AT variability, even after adjustment for FVIIa levels, ${ }^{17}$ thus addressing the interest on the complex and still controversial link between plasma lipids and coagulation.
On the basis of such premises, to investigate more in depth the relationship between FVIIa-AT and plasma lipids, we performed a new series of analyses evaluating a broad apolipoprotein profile, including apolipoprotein A-I (ApoAI), B (ApoB), C-III (ApoC-III) and E (ApoE), in the original VHS population cohort previously assessed for FVIIa-AT.

\section{Materials and Methods}

\section{Study Population}

This study was performed within the framework of the VHS, a regional survey aimed to look for new CAD risk factors in subjects with angiographic documentation of their coronary vessels. ${ }^{19}$ Briefly, all the subjects in the VHS are required to have no history of any acute illness in the month preceding the enrolment. CAD patients with acute coronary syndrome were excluded from this study. Subjects with severe renal failure (estimated glomerular filtration rate [eGFR] $<30 \mathrm{~mL} / \mathrm{min}$ ) and those with severe hepatic impairment (clinically defined diagnosis of liver cirrhosis) were also excluded from this study. The study design is summarized in -Supplementary Fig. S1, available in the online version. Within the original study population not taking any anticoagulant drugs who have been assessed for FVIIa-AT levels, ${ }^{17}$ we selected the 666 subjects for whom plasma samples for apolipoprotein assays were available (75.4\% males, mean age: $61.1 \pm 10.9$ years). One hundred and thirty-one subjects having completely normal coronary arteries (undergoing coronary angiography for reasons other than $C A D$, mainly valvular heart disease) were used as controls (CAD-free group). These subjects had also no history, or clinical or instrumental evidence of atherosclerosis outside the coronary bed. Five hundred and thirty-five subjects had angiographically proven CAD (CAD group), with at least one of the major epicardial coronary arteries (left anterior descending, circumflex and right) affected with $\geq 1$ significant stenosis ( $\geq 50 \%$ lumen reduction). All the CAD patients were newly diagnosed at time of enrolment, that is, at time of coronary angiography. The angiograms were assessed blind by two cardiologists who were unaware that the patients were to be included in this study.

All participants came from the same geographical area of northern Italy. At the time of blood sampling, a complete clinical history was collected, as well as data about drug therapies. The study complies with the Declaration of Helsinki and was approved by the Ethic Committee of our institution (Azienda Ospedaliera Universitaria Integrata, Verona, Italy). A written informed consent was obtained from all the participants.

\section{Biochemical Analysis}

Samples of venous blood were drawn from each subject, after an overnight fast, at the time of enrolment before coronary angiography. Serum lipids, as well as other CAD risk factors, including high-sensitivity C-reactive protein (hs-CRP), were determined as previously described. ${ }^{17,19}$ Very low-density lipoprotein (VLDL) cholesterol was calculated by subtracting from total cholesterol the concentration of HDL and lowdensity lipoprotein (LDL) cholesterol. The four variables 
version of the Modification of Diet in Renal Disease equation was used to estimate the GFR from serum creatinine levels. ${ }^{20}$

\section{Apolipoprotein Assays}

ApoA-I, ApoB and ApoE were measured by commercially available nephelometric immunoassays, as previously described. ${ }^{21}$ ApoC-III concentration was measured using an automated turbidimetric immunoassay (Wako Pure Chemical Industries). Intra-assay coefficients of variation (CVs) were $1.84,2.02$ and $1.98 \%$ on three pools of control sera with low, medium and high concentrations of ApoCIII, respectively; inter-assay CVs were $4.4,3.4$ and $2.29 \%$ for low, medium and high concentration, respectively. ${ }^{22,23}$

\section{FVIla-AT and FVIla Assays}

The concentration of FVIIa-AT was measured by enzymelinked immunosorbent assay (Asserachrom VIIa-AT, Diagnostica Stago, Asnieres, France) on frozen citrate plasma samples, never thawed before this study. Venous blood samples collected at the time of enrolment were centrifuged, stored in $0.5 \mathrm{~mL}$ aliquots and frozen at $-80^{\circ} \mathrm{C}$ within 1 hour after sample collection. Plasma samples were thawed in a water bath at $37^{\circ} \mathrm{C}$ for 5 minutes before FVIIa-AT assay. All testing was performed in duplicate. The intra- and interassay CVs were $<5 \%{ }^{17}$

FVIIa was assessed with a kit utilizing a soluble recombinant truncated TF that is selectively deficient in promoting FVII activation but retains recombinant FVIIa (rFVIIa) cofactor function, thus allowing direct quantification of FVIIa in plasma (Staclot VIIa-rTF, Diagnostica Stago). Values were expressed in milliunits per millilitre, 30 such units being equivalent to $1 \mathrm{ng}$ of FVIIa. The standard was a rFVIIa supplied with the kit. The intra- and inter-assay CVs were 7.8 and $6.4 \%$, respectively. ${ }^{21}$ Plasma samples for FVIIa assay were available for 291 subjects (43.7\%).

\section{Single Nucleotide Polymorphisms and Genotyping}

Genomic deoxyribonucleic acid (DNA) was prepared from whole blood samples by phenol-chloroform extraction.

The rs964184 C/G polymorphism is located on chromosome 11q23.3, tagging ZNF259, APOA5-APOA4-APOC3-APOA1 gene region. Genome-wide association studies (GWAS) proved that the $G$ allele is associated with high levels of triglycerides and LDL cholesterol, low levels of HDL cholesterol and an increased risk of CAD. ${ }^{24-26}$ rs964184 single nucleotide polymorphism (SNP) was genotyped using Taqman platform. DNA samples for rs964184 analysis were available for 540 subjects $(81.0 \%)$.

Polymorphisms in the APOE gene, rs429358 (Cys112Arg) and rs7412 (Arg158Cys), encode three common alleles, $\varepsilon 2$ (Cys122 and Cys158), $\varepsilon 3$ (Cys112 and Arg158) and $\varepsilon 4$ (Arg112 and Arg158), which combine to form six genotypes, $\varepsilon 2 / \varepsilon 2$, $\varepsilon 2 /$ $\varepsilon 3, \varepsilon 2 / \varepsilon 4, \varepsilon 3 / \varepsilon 3, \varepsilon 3 / \varepsilon 4$ and $\varepsilon 4 / \varepsilon 4$. APOE $\varepsilon 2 / \varepsilon 3 / \varepsilon 4$ polymorphisms are among the most investigated gene variants being a key regulator of lipoprotein metabolism. ${ }^{27,28}$ APOE polymorphisms were genotyped according to a previously described multi-locus assay ${ }^{29}$ and data were available for 495 subjects $(74.3 \%)$.

\section{Statistics}

All calculations were performed using the IBM SPSS 20.0 (IBM Inc., Armonk, New York, United States) statistical package.

Distributions of continuous variables in groups were expressed as means \pm standard deviations. Skewed variables, including FVIIa-AT, FVIIa, hs-CRP, VLDL cholesterol, triglyceride, ApoC-III and ApoE, were logarithmically transformed and then geometric means with 95\% confidence interval were reported. Quantitative data were assessed using the Student's $t$-test or by analysis of variance, with polynomial contrast for linear trend when indicated. Qualitative data were analysed with the chi-square test and with chi-square for linear trend analysis when indicated. The frequencies of the genotypes associated with each of the assessed polymorphisms were compared by using the chi-square test with the values predicted on the basis of the Hardy-Weinberg equilibrium.

Significant associations between FVIIa-AT plasma concentration and lipid parameters (i.e. traditional plasma lipids and apolipoproteins) were evaluated at first by Pearson's correlation coefficient $(R)$ in the whole study population, as well as in either males or females and in either CAD or CAD-free subgroups. To assess the independent predictors of FVIIa-AT levels, all the variables showing an association with the FVIIa-AT complex at univariate analysis were included in an adjusted regression model. Further adjustments were performed including in the regression models sex, age, body mass index (BMI), eGFR, smoking status, CAD diagnosis, lipid-lowering therapy and FVIIa. Taking into account the low number of subjects for whom FVIIa levels were available $(n=291)$, data of FVIIa were added in a separate adjusted regression model.

A value of $p<0.05$ was considered statistically significant.

\section{Results}

Clinical and laboratory characteristics of the study population are reported in - Table $\mathbf{1}$. As previously observed, ${ }^{17}$ no difference in FVIIa-AT levels was found between CAD and CAD-free subjects ( - Table $\mathbf{1}$ ). As regards plasma lipids and apolipoproteins, HDL cholesterol and ApoA-I were lower while triglyceride and ApoC-III were higher in patients with CAD than in CADfree subjects, although with a substantial overlap in plasma levels between cases and controls (-Table 1). In the whole study population, several significant direct correlations were found between FVIIa-AT and lipid variables. Total and HDL cholesterol, triglycerides, ApoA-I, ApoC-III and ApoE correlated directly with FVIIa-AT levels ( - Table 2 ). The association with ApoC-III was the strongest with high statistical significance $\left(R=0.235, p=7.7 \times 10^{-10} ;\right.$ - Fig. 1$)$ and was the only confirmed association in all the sub-group analyses (i.e. males and females, CAD and CAD-free; - Table 2). Including total and HDL cholesterol, triglycerides, ApoA-I, ApoC-III and ApoE (i.e. the variables showing an association with the FVIIa-AT complex at univariate analysis) in a regression model for FVIIa-AT variability, only ApoC-III remained associated with FVIIa-AT (-Table 3). Such association was confirmed after adjustment for sex, age, CAD diagnosis, BMI, eGFR, smoking status and lipid-lowering therapies (standardized $\beta$-coefficient $=0.158$, $p=0.011$; - Table 3). Taking into account that ApoC-III levels 
Table 1 Clinical and laboratory characteristics of the study population, considered as a whole or divided in sub-groups with or without coronary artery disease (CAD)

\begin{tabular}{|c|c|c|c|c|}
\hline & $\begin{array}{l}\text { Total population } \\
(n=666)\end{array}$ & $\begin{array}{l}\text { CAD-free } \\
(n=131)\end{array}$ & $\begin{array}{l}\text { CAD } \\
(n=535)\end{array}$ & $p$-Value ${ }^{a}$ \\
\hline Age (y) & $61.1 \pm 10.9$ & $60.4 \pm 11.9$ & $61.3 \pm 10.7$ & NS \\
\hline Males (\%) & 75.4 & 69.5 & 76.8 & NS \\
\hline BMI $\left(\mathrm{kg} / \mathrm{m}^{2}\right)$ & $26.6 \pm 3.8$ & $26.0 \pm 3.6$ & $26.8 \pm 3.8$ & 0.042 \\
\hline Diabetes (\%) & 19.0 & 8.1 & 21.6 & 0.001 \\
\hline Hypertension (\%) & 64.1 & 45.3 & 68.7 & $<0.001$ \\
\hline Smoke (\%) & 63.8 & 43.7 & 68.5 & $<0.001$ \\
\hline $\mathrm{eGFR}(\mathrm{mL} / \mathrm{min})^{\mathrm{b}}$ & $73.7 \pm 19.9$ & $74.2 \pm 21.0$ & $73.5 \pm 19.7$ & NS \\
\hline hs-CRP (mg/L) & $\begin{array}{l}4.23 \\
(3.78-4.73)\end{array}$ & $\begin{array}{l}2.33 \\
(1.84-2.94)\end{array}$ & $\begin{array}{l}4.98 \\
(4.40-5.64)\end{array}$ & $<0.001$ \\
\hline Lipid-lowering therapy (\%) & 25.4 & 8.4 & 29.5 & $<0.001$ \\
\hline Total cholesterol (mmol/L) & $5.18 \pm 1.10$ & $5.21 \pm 1.04$ & $5.17 \pm 1.11$ & NS \\
\hline LDL cholesterol (mmol/L) & $3.41 \pm 0.94$ & $3.34 \pm 0.95$ & $3.42 \pm 0.93$ & NS \\
\hline HDL cholesterol (mmol/L) & $1.20 \pm 0.33$ & $1.38 \pm 0.41$ & $1.16 \pm 0.29$ & $<0.001$ \\
\hline VLDL cholesterol (mmol/L) & $\begin{array}{l}0.67 \\
(0.65-0.69)\end{array}$ & $\begin{array}{l}0.54 \\
(0.50-0.58)\end{array}$ & $\begin{array}{l}0.71 \\
(0.68-0.73)\end{array}$ & $<0.001$ \\
\hline Triglyceride (mmol/L) & $\begin{array}{l}1.58 \\
(1.53-1.64)\end{array}$ & $\begin{array}{l}1.28 \\
(1.19-1.36)\end{array}$ & $\begin{array}{l}1.67 \\
(1.61-1.73)\end{array}$ & $<0.001$ \\
\hline ApoA-I (g/L) & $1.28 \pm 0.26$ & $1.36 \pm 0.30$ & $1.26 \pm 0.25$ & $<0.001$ \\
\hline ApoB $(\mathrm{g} / \mathrm{L})$ & $1.04 \pm 0.28$ & $1.01 \pm 0.25$ & $1.05 \pm 0.28$ & NS \\
\hline ApoC-III (mg/dL) & $\begin{array}{l}10.5 \\
(10.2-10.8)\end{array}$ & $\begin{array}{l}9.9 \\
(9.3-10.5)\end{array}$ & $\begin{array}{l}10.6 \\
(10.3-11.0)\end{array}$ & 0.038 \\
\hline ApoE (g/L) & $\begin{array}{l}0.037 \\
(0.035-0.038)\end{array}$ & $\begin{array}{l}0.038 \\
(0.035-0.039)\end{array}$ & $\begin{array}{l}0.036 \\
(0.035-0.037)\end{array}$ & NS \\
\hline FVIla-AT (pM) & $\begin{array}{l}84.7 \\
(81.5-88.0)\end{array}$ & $\begin{array}{l}85.2 \\
(77.4-93.9)\end{array}$ & $\begin{array}{l}84.5 \\
(81.1-88.1)\end{array}$ & NS \\
\hline FVIIla $(\mathrm{mU} / \mathrm{mL})^{\mathrm{c}}$ & $\begin{array}{l}46.9 \\
(43.8-50.1)\end{array}$ & $\begin{array}{l}46.1 \\
(40.0-53.0)\end{array}$ & $\begin{array}{l}47.2 \\
(43.7-50.9)\end{array}$ & NS \\
\hline
\end{tabular}

Abbreviations: Apo, apolipoprotein; BMI, body mass index; eGFR, estimated glomerular filtration rate; FVIla-AT, factor VII-anti-thrombin; HDL, highdensity lipoprotein; hs-CRP, high-sensitivity C-reactive protein; LDL, low-density lipoprotein; MDRD, Modification of Diet in Renal Disease; NS, not significant; VLDL, very low-density lipoprotein.

${ }^{a}$ By $t$-test or chi-square test, when appropriate.

${ }^{\mathrm{b}}$ By MDRD formula.

'Data were available for 291 subjects (76 CAD-free and 215 CAD).

correlated with all plasma lipid parameters (- Supplementary Table S1, available in the online version), a further regression model including all plasma lipids and apolipoproteins was performed and found that ApoC-III remained associated with FVIIa-AT also by this analysis (standardized $\beta$-coefficient $=0.209, p=0.004$ ). The strong correlation between ApoC-III and FVIIa-AT was confirmed in the sub-group of subjects not taking lipid-lowering therapies at enrolment $(n=497$; $\left.R=0.251, p=1.3 \times 10^{-8}\right)$, even in full-adjusted regression model (standardized $\beta$-coefficient $=0.183, p=0.007$ ). Considering the potential influence of smoking status on both TRLs metabolism and TF-FVIIa pathway, analyses according to smoking status were also performed. The strong direct correlation between ApoC-III and FVIIa-AT plasma levels was con- firmed in both smokers $\left(R=0.221, p=7.0 \times 10^{-6}\right)$ and nonsmokers $\left(R=0.269, p=3.6 \times 10^{-5}\right)$.

Within the sub-group of subjects with available data of FVIIa ( $n=291)$, ApoC-III plasma concentration correlated directly also with FVIIa levels $\left(R=0.291, p=4.4 \times 10^{-7}\right)$. In an adjusted regression model, both ApoC-III (standardized $\beta$-coefficient $=0.178, p=0.001$ ) and FVIIa (standardized $\beta$-coefficient $=0.479, p<0.001)$ remained significant predictors of FVIla-AT variability (- Supplementary Table S2, available in the online version).

Stratifying the study population on the basis of ApoC-III plasma concentration, FVIIa-AT levels increased progressively from the lowest to the highest quartile (-Fig. 2 and -Supplementary Table S3, available in the online version) 
Table 2 Correlations between activated factor VII-anti-thrombin complex (FVIla-AT) and plasma lipids and apolipoproteins, in the whole study population and in the different sub-groups on the basis of coronary artery disease (CAD) diagnosis and gender

\begin{tabular}{|c|c|c|c|c|c|c|c|c|c|c|}
\hline & \multicolumn{2}{|c|}{$\begin{array}{l}\text { Whole study } \\
\text { population } \\
(n=666)\end{array}$} & \multicolumn{2}{|c|}{$\begin{array}{l}\text { CAD-free } \\
(n=131)\end{array}$} & \multicolumn{2}{|c|}{$\begin{array}{l}\text { CAD } \\
(n=535)\end{array}$} & \multicolumn{2}{|c|}{$\begin{array}{l}\text { Males } \\
(n=502)\end{array}$} & \multicolumn{2}{|c|}{$\begin{array}{l}\text { Females } \\
(n=164)\end{array}$} \\
\hline & $R$ & $p$ & $R$ & $p$ & $R$ & $p$ & $R$ & $p$ & $R$ & $p$ \\
\hline Total cholesterol & 0.081 & 0.041 & 0.234 & 0.008 & 0.040 & NS & 0.098 & 0.030 & -0.006 & NS \\
\hline LDL cholesterol & 0.040 & NS & 0.161 & NS & 0.008 & NS & 0.050 & NS & 0.028 & NS \\
\hline HDL cholesterol & 0.117 & 0.005 & 0.170 & NS & 0.091 & 0.049 & 0.101 & 0.034 & 0.035 & NS \\
\hline VLDL cholesterol & 0.058 & NS & 0.145 & NS & 0.042 & NS & 0.045 & NS & 0.122 & NS \\
\hline Triglyceride & 0.110 & 0.005 & 0.218 & 0.014 & 0.091 & 0.038 & 0.120 & 0.008 & 0.121 & NS \\
\hline ApoA-I & 0.158 & $<0.001$ & 0.132 & NS & 0.168 & $<0.001$ & 0.183 & $<0.001$ & 0.035 & NS \\
\hline ApoB & -0.020 & NS & 0.107 & NS & -0.023 & NS & -0.021 & NS & 0.009 & NS \\
\hline ApoC-III & 0.235 & $<0.001$ & 0.336 & $<0.001$ & 0.211 & $<0.001$ & 0.244 & $<0.001$ & 0.187 & 0.016 \\
\hline ApoE & 0.125 & 0.001 & 0.090 & NS & 0.135 & 0.002 & 0.161 & $<0.001$ & -0.024 & NS \\
\hline
\end{tabular}

Abbreviations: Apo, apolipoprotein; HDL, high-density lipoprotein; LDL, low-density lipoprotein; NS, not significant; VLDL, very low-density lipoprotein. Note: Significant correlations are reported in bold.

and such trend was confirmed in all the main sub-group analyses (-Fig. 2), including those in smoker and nonsmoker sub-populations (- Supplementary Fig. S2, available in the online version). As expected, subjects with high ApocIII levels had an unfavourable lipid profile (- Supplementary Table S3, available in the online version).

Considering the strong direct correlation of ApoC-III with triglycerides $\left(R=0.559, \quad p=2.6 \times 10^{-54}\right)$ and ApoE $\left(R=0.290, p=2.2 \times 10^{-14}\right)$, which are all parameters characterizing TRLs, we performed a further analysis stratifying the study population on the basis of triglycerides, ApoC-III and ApoE median values. All the sub-groups having ApoC-III above the median plasma concentration had increased levels of FVIIAT rather than those with low ApoC-III, independent of both triglycerides and ApoE plasma concentration (- Fig. 3).

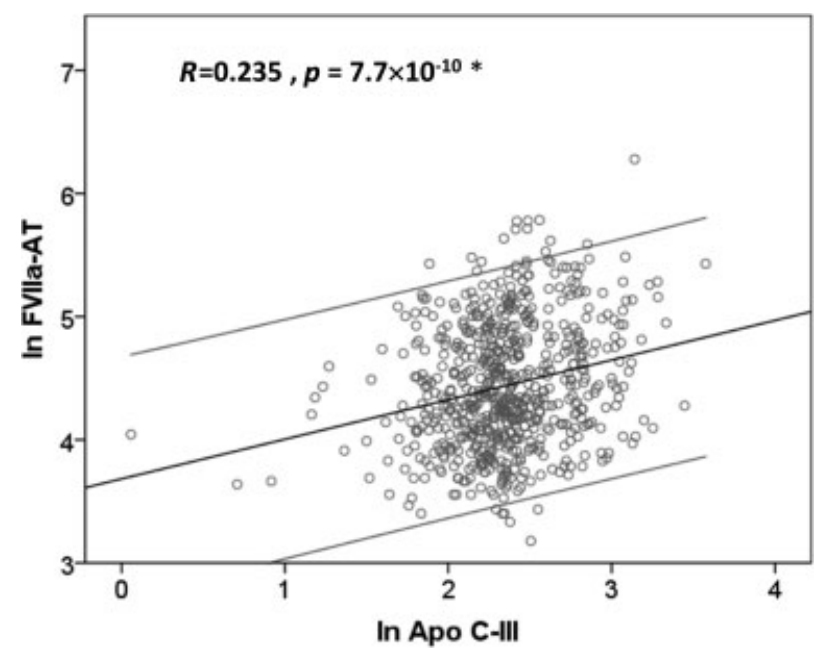

Fig. 1 Correlation between plasma concentrations of activated factor VII-anti-thrombin complex (FVIla-AT) and apolipoprotein C-III (ApoC-III) in the whole study population. ${ }^{*}$ Pearson's correlation test.
We investigated also genetic components supporting the association between ApoC-III and FVII-AT. Within the study population, 540 subjects were genotyped for rs 964184 polymorphism, which tags APOA5-APOA4-APOC3-APOA1 locus and has been linked by GWAS with both dyslipidaemia and CAD. The genotype distribution was consistent with the HardyWeinberg equilibrium. As expected, the carriers of the minor allele $(G)$ were more represented among CAD and had an

Table 3 Linear regression analysis for variability of activated factor VII-anti-thrombin complex (FVIla-AT) plasma levels

\begin{tabular}{|l|l|l|l|l|}
\hline & \multicolumn{2}{|l|}{ Model 1 } & \multicolumn{2}{l|}{ Model 2 } \\
\cline { 2 - 5 } & $\begin{array}{l}\text { Standardized } \\
\beta \text {-coefficient }\end{array}$ & $p$-Value & $\begin{array}{l}\text { Standardized } \\
\beta \text {-coefficient }\end{array}$ & $p$-Value \\
\hline $\begin{array}{l}\text { Total } \\
\text { cholesterol }\end{array}$ & -0.087 & 0.076 & -0.060 & 0.264 \\
\hline $\begin{array}{l}\mathrm{HDL} \\
\text { cholesterol }\end{array}$ & 0.108 & 0.113 & 0.065 & 0.395 \\
\hline Triglyceride & 0.048 & 0.426 & 0.047 & 0.465 \\
\hline ApoA-I & 0.053 & 0.422 & 0.114 & 0.108 \\
\hline ApoC-III & $\mathbf{0 . 2 0 0}$ & $\mathbf{0 . 0 0 1}$ & $\mathbf{0 . 1 5 8}$ & $\mathbf{0 . 0 1 1}$ \\
\hline ApoE & 0.066 & 0.135 & 0.028 & 0.550 \\
\hline
\end{tabular}

Abbreviations: Apo, apolipoprotein; BMI, body mass index; CAD, coronary artery disease; eGFR, estimated glomerular filtration rate; $\mathrm{HDL}$, high-density lipoprotein.

Note: FVIla-AT was considered as dependent variable, while all plasma lipids and apolipoproteins showing a significant correlation at univariate analysis were included as independent variables (Model 1). The regression model was then adjusted for sex, age, CAD diagnosis, BMI, renal function and lipid-lowering therapies at enrolment (Model 2). Significant associations are reported in bold.

Model 1: Total cholesterol, HDL cholesterol, triglyceride, ApoA-I, ApoC-III and $A p o E$ as independent variables.

Model 2: Model 1 plus sex, age, CAD diagnosis, BMI, eGFR, smoking status and lipid-lowering therapies at enrolment as independent variables. 
Whole study population $(n=666)$

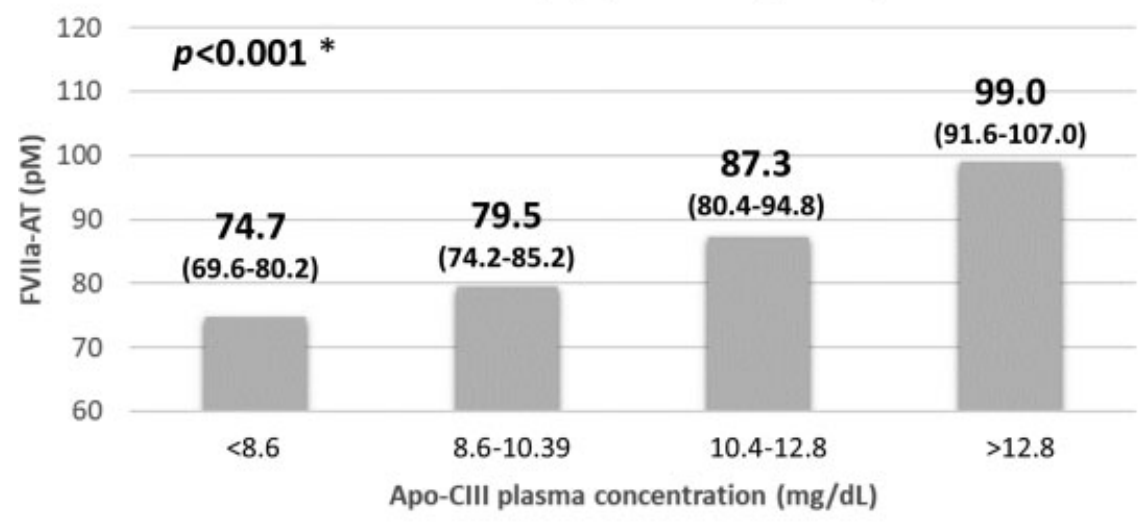

CAD-free $(n=131)$

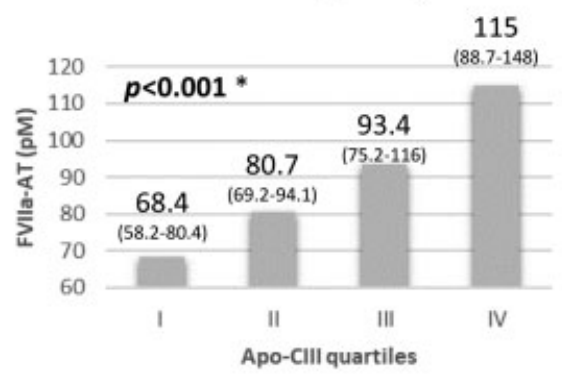

Males $(n=502)$

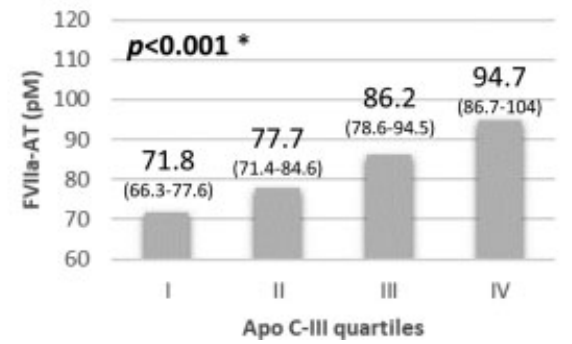

CAD $(n=535)$

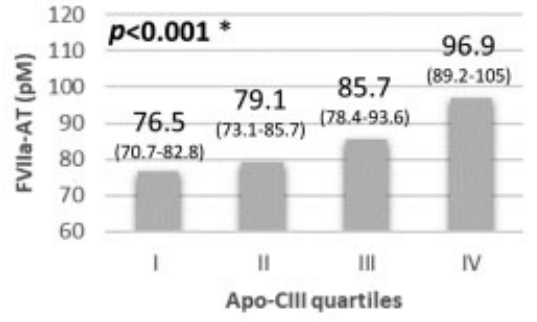

Females $(n=164)$

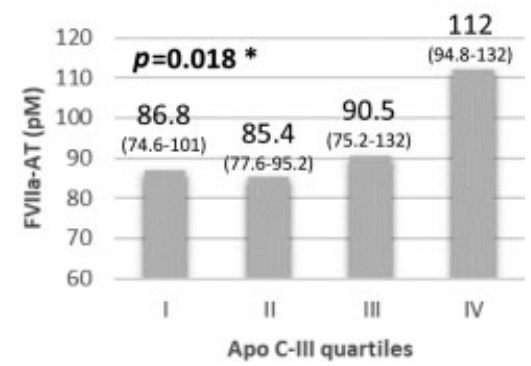

Fig. 2 Activated factor VII-anti-thrombin complex (FVIla-AT) levels according to apolipoprotein C-III (ApoC-III) plasma concentration quartiles in the whole study population, in subjects with or without coronary artery disease (CAD) and in male and female sub-groups. *Analysis of variance (ANOVA) with polynomial contrasts for linear trend.

unfavourable lipid profile (-Table 4). While no significant difference was found for ApoA-I, ApoB and ApoE, the carriers of the $G$ allele had higher ApoC-III plasma concentration and also higher FVIIa-AT levels (-Fig. 4). Within the genotyped population, data on FVIIa were available for 253 subjects and $G$ allele carriers had increased levels of FVIIa ( - Table 4 ). On the other hand, APOE $\varepsilon 2 / \varepsilon 3 / \varepsilon 4$ genotypes were not related with FVIIa-AT levels, while-as expected-the associations with ApoE, triglycerides and VLDL cholesterol were confirmed (-Supplementary Table S4, available in the online version).

\section{Discussion}

In this study, we show that (1) ApoC-III has the strongest association with FVIIa-AT among plasma lipid parameters, and (2) carriers of the minor allele of rs964184 gene poly- morphism, tagging $A P O C 3$ locus and known to influence ApoC-III plasma concentration and cardiovascular risk, ${ }^{24-26}$ had FVIIa-AT levels higher than non-carriers. Thereby, these results suggest a link between ApoC-III and the TF-related initiation phase of the blood clotting cascade.

ApoC-III is a crucial player of TRLs metabolism and is recognized as a key determinant of cardiovascular risk by correlating with an unfavourable lipoprotein metabolism. ${ }^{30,31}$ ApoC-III, synthesized mainly in the liver, resides mostly on the surface of VLDLs, but also on LDLs and HDLs, and can reduce the clearance of ApoB lipoproteins from the circulation by interfering with their binding to hepatic ApoB/ApoE receptors. ${ }^{32}$ Moreover, ApoC-III inhibits the action of lipoprotein lipase to hydrolyze lipoprotein triglycerides. Finally, ApoC-III contributes to the formation of TRLs in liver cells, stimulating the secretion of VLDL. ${ }^{32}$ GWAS have identified polymorphisms 


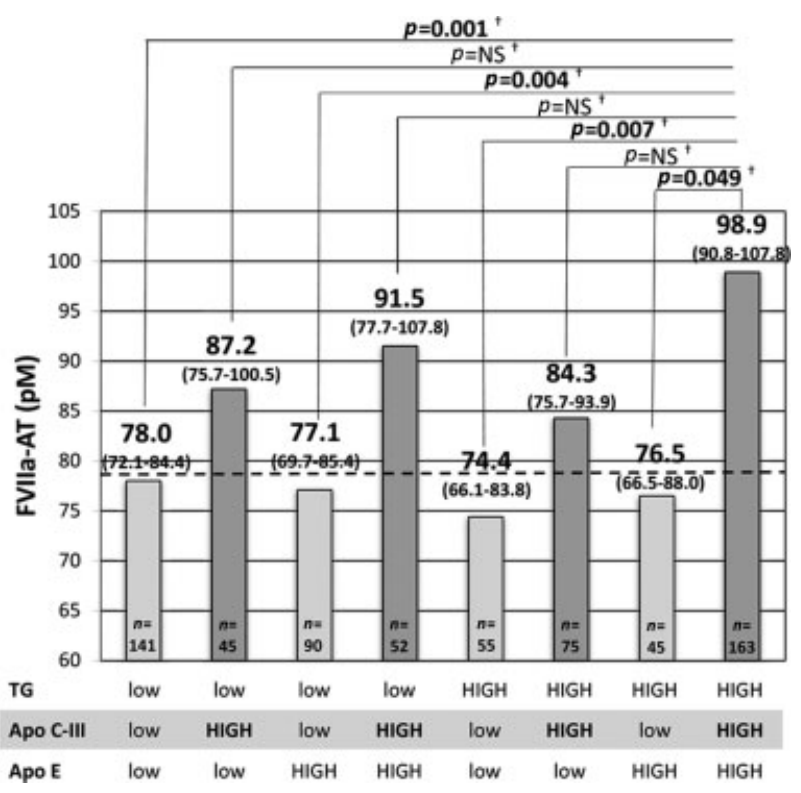

Fig. 3 Activated factor VII-anti-thrombin complex (FVlla-AT) levels according to triglyceride (TG), apolipoprotein C-III (ApoC-III) and apolipoprotein $\mathrm{E}$ (ApoE) plasma concentrations. ${ }^{* *}$ The whole study population has been stratified according the median levels of TG $(1.56 \mathrm{mmol} / \mathrm{L})$, ApoC-III $(10.4 \mathrm{mg} / \mathrm{dL})$ and ApoE $(0.037 \mathrm{~g} / \mathrm{L})$. Therefore, eight sub-groups were obtained, each characterized by a combination of high or low plasma concentration of TG, ApoC-III and ApoE. The horizontal dotted line indicates the median level of FVIla-AT in the whole study population (78.5 pM). †Analysis of variance (ANOVA) with Tukey's post hoc comparison.

tagging APOC3 that strongly associated with both dyslipidaemia and CVD, according with the hypothesis of an increased cardiovascular risk mediated by TRLs. ${ }^{24-26}$ Loss-of-function mutations of the APOC3 gene have been associated with a decreased risk of CVD paving the way to ApoC-III-lowering therapies as innovative approaches to reduce cardiovascular risk. ${ }^{33}$ Volanesorsen-an anti-ApoC-III antisense oligonucleotide-has been demonstrated in phase II clinical trials to be potentially a new powerful tool for the therapy of hypertriglyceridaemia. ${ }^{34}$ Noteworthy, the mechanisms linking TRLs (including ApoC-III-rich lipoproteins) and CVD go beyond the traditional view of atherosclerotic plaque development due to lipid accumulation and may include excessive free fatty acid release and production of pro-inflammatory cytokines, which in turn could increase TF expression, as well as stimulation of coagulation factors and impairment of fibrinolysis. ${ }^{3}$

In this article, we add a further tile to the mosaic of pleiotropic effects of ApoC-III, emphasizing its potential procoagulant capability, which has previously been addressed by our group. ${ }^{22,23}$ We originally showed that increased ApoCIII concentrations correlated with an amplified plasma endogenous thrombin generation. ${ }^{22}$ Then, we found that high ApoC-III levels were associated with an increase of FII coagulant activity to an extent comparable with the carriership of the A allele of $F 2$ 20210G > A gene polymorphism. ${ }^{23}$ We also observed a direct correlation of ApoC-III plasma concentration with both FVIIa and FV coagulant activities. Finally, high levels of ApoC-III were related with an enhanced activated FXa generation. ${ }^{23}$
The results of this study, showing a strong direct correlation between ApoC-III and FVIIa-AT, appear consistent with earlier findings (-Supplementary Fig. $\mathbf{3 3}$, available in the online version). The correlation of ApoC-III with FVIIa-AT suggests increased intravascular amounts of TF-FVIIa complex in subjects with high ApoC-III plasma concentrations. An increased exposure of decrypted TF could be hypothesized, leading to an enhanced FVII activation and consequently to a greater FXa generation..$^{23}$ The extrinsic pathway potentiation would be reflected in an amplified common pathway, resulting into increased FV and FII coagulant activities ${ }^{23}$ and thrombin generation. ${ }^{22}$

In our previous work, both triglycerides and HDL cholesterol correlated directly with FVIIa-AT, ${ }^{17}$ but in this analysis such correlations were no longer evident after adjustment for ApoC-III. ApoC-III is expressed on both TRLs and HDLs and recent data support that it may not only characterize the harmful effects of TRLs but also may adversely affect the antiatherogenic properties of HDL. ${ }^{35,36}$ This result prompts that ApoC-III may be a key player of pro-coagulant effects associated to both TRLs and HDLs. Considering all the parameters characterizing TRLs which were available in our study (i.e. triglyceride, ApoC-III and ApoE), only an elevated ApoC-III concentration was unvaryingly associated with high FVIIa-AT levels. Our results are consistent with previous works showing the pro-coagulant properties of VLDL, which typically express ApoC-III. ${ }^{9} 10$ Thrombin generation sustained by VLDLs was found to be 19.4-fold greater than that sustained by HDLs and 11.7-fold greater than that sustained by LDLs. ${ }^{9} 10$ VLDLs support all the components of the extrinsic coagulation pathway ${ }^{10}$ and can both stimulate TF expression and enhance TF-independent FVII activation. ${ }^{37}$ VLDLs are functionally characterized by ApoC-III expression. However, it is not known if ApoC-III might participate in VLDL microdomains with distinct local lipid compositions able to support/enhance TF-FVIIa interaction. It may be also speculated that the rise of FVIIa-AT is secondary to the TRLs-related increase of FVIIa levels, whose concentration has been found to be the main determinant of FVIIa-AT in plasma. ${ }^{16}$ However, in our analysis the association between ApoC-III and FVIIa-AT was independent of FVIIa, thus suggesting that ApoC-III may influence expression and/or activity of TF rather than merely FVIla levels. It is worthy to note that in a recent study high FVIIa-AT levels were related with postprandial lipaemia. ${ }^{38}$ Since post-prandial lipaemia is characterized by high concentrations of ApoC-III, ${ }^{39}$ such observation appears consistent with our findings. Moreover, considering the rapid change in levels observed within short time intervals ( $<6$ hours), ${ }^{38}$ it could suggest involvement of release/activation processes rather than biosynthesis of new TF and FVII molecules.

Our hypothesis of a pro-coagulant role of ApoC-III is strengthened by the analysis of rs964184 polymorphism, which tags the gene cluster containing the $A P O C 3$ locus and has been linked by GWAS with both dyslipidaemia and CAD. ${ }^{24-26}$ This variant exerts multiple functional effects on the expression of the gene cluster. ${ }^{40}$ The higher levels of FVIIa-AT found in carriers of the risk allele $G$ further supports 
Table 4 Clinical and laboratory characteristics, including plasma lipids and activated factor VII-anti-thrombin complex (FVIIa-AT) levels, according rs964184 genotype (data available for 540 subjects)

\begin{tabular}{|c|c|c|c|c|}
\hline & rs964184 polyr & & & $p$-Value ${ }^{a}$ \\
\hline & $\begin{array}{l}\text { CC } \\
(n=400)\end{array}$ & $\begin{array}{l}\text { CG } \\
(n=127)\end{array}$ & $\begin{array}{l}\text { GG } \\
(n=13)\end{array}$ & \\
\hline Age $(y)$ & $60.5 \pm 11.2$ & $62.1 \pm 9.5$ & $65.0 \pm 10.0$ & NS \\
\hline Males (\%) & 76.3 & 76.4 & 76.9 & NS \\
\hline CAD diagnosis (\%) & 80.3 & 86.6 & 92.3 & 0.058 \\
\hline BMI $\left(\mathrm{kg} / \mathrm{m}^{2}\right)$ & $26.5 \pm 3.7$ & $26.9 \pm 3.9$ & $26.5 \pm 5.3$ & NS \\
\hline Lipid-lowering therapy (\%) & 24.8 & 28.3 & 23.1 & NS \\
\hline Total cholesterol (mmol/L) & $5.19 \pm 0.98$ & $5.24 \pm 1.35$ & $5.25 \pm 1.02$ & NS \\
\hline LDL cholesterol (mmol/L) & $3.34 \pm 0.85$ & $3.69 \pm 1.10$ & $3.54 \pm 0.79$ & 0.007 \\
\hline HDL cholesterol (mmol/L) & $1.20 \pm 0.33$ & $1.17 \pm 0.27$ & $1.15 \pm 0.20$ & NS \\
\hline VLDL cholesterol (mmol/L) & $\begin{array}{l}0.66 \\
(0.63-0.69)\end{array}$ & $\begin{array}{l}0.71 \\
(0.66-0.76)\end{array}$ & $\begin{array}{l}0.89 \\
(0.72-1.11)\end{array}$ & 0.011 \\
\hline Triglyceride (mmol/L) & $\begin{array}{l}1.57 \\
(1.50-1.64)\end{array}$ & $\begin{array}{l}1.69 \\
(1.57-1.82)\end{array}$ & $\begin{array}{l}2.18 \\
(1.85-2.57)\end{array}$ & 0.005 \\
\hline ApoA-I (g/L) & $1.26 \pm 0.26$ & $1.26 \pm 0.26$ & $1.27 \pm 0.22$ & NS \\
\hline ApoB $(g / L)$ & $1.05 \pm 0.26$ & $1.05 \pm 0.32$ & $1.02 \pm 0.35$ & NS \\
\hline ApoC-III (mg/dL) & $\begin{array}{l}10.5 \\
(10.1-10.8)\end{array}$ & $\begin{array}{l}10.8 \\
(10.1-11.5)\end{array}$ & $\begin{array}{l}13.9 \\
(11.6-16.7)\end{array}$ & 0.033 \\
\hline ApoE (g/L) & $\begin{array}{l}0.036 \\
(0.035-0.038)\end{array}$ & $\begin{array}{l}0.036 \\
(0.034-0.038)\end{array}$ & $\begin{array}{l}0.038 \\
(0.028-0.052)\end{array}$ & NS \\
\hline FVIIa-AT (pM) & $\begin{array}{l}81.4 \\
(77.7-85.4)\end{array}$ & $\begin{array}{l}89.9 \\
(83.0-97.4)\end{array}$ & $\begin{array}{l}97.7 \\
(75.7-126.0)\end{array}$ & 0.018 \\
\hline FVIIla $(\mathrm{mU} / \mathrm{mL})^{\mathrm{b}}$ & $\begin{array}{l}43.8 \\
(39.9-48.0)\end{array}$ & $\begin{array}{l}53.4 \\
(45.9-62.1)\end{array}$ & $\begin{array}{l}51.7 \\
(42.0-63.7)\end{array}$ & 0.042 \\
\hline
\end{tabular}

Abbreviations: Apo, apolipoprotein; ANOVA, analysis of variance; BMI, body mass index; CAD, coronary artery disease; HDL, high-density lipoprotein; LDL, low-density lipoprotein; NS, not significant; VLDL, very low-density lipoprotein.

${ }^{a}$ By ANOVA with polynomial contrasts for linear trend or chi-square test for linear trend, when appropriate.

${ }^{b}$ Data were available for 253 subjects (CC, $n=188$; CG, $n=56$; GG, $n=7$ ).

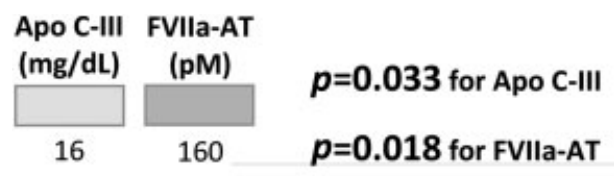

$14 \quad 140$

12120

10

8

6
$120 \quad 10.5$ (10.1-10.8)

100

80

60

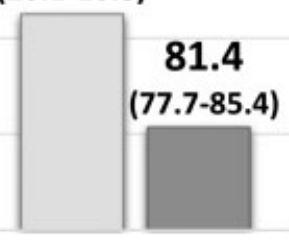

CC ( $n=400)$
10.8

(10.1-11.5)

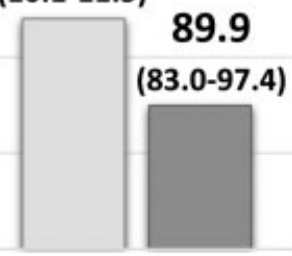

CG

( $n=127$ )
13.9

(11.6-16.7)

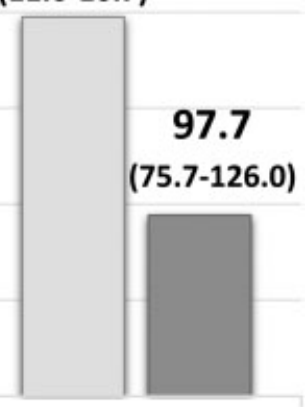

GG

( $n=13$ )

Fig. 4 Plasma concentrations of apolipoprotein C-III (ApoC-III) and activated factor VII-anti-thrombin complex (FVIIa-AT) according to rs964184 genotypes. 
a link between ApoC-III and extrinsic coagulation pathway. Noteworthy, another key gene variant in TRLs metabolism, that is, the $A P O E \varepsilon 2 / \varepsilon 3 / \varepsilon 4$ polymorphism, did not associate with FVIIa-AT levels, consistently with the analysis of FVIIaAT on the basis of triglyceride, ApoC-III and ApoE levels (-Fig. 3). Up to now GWAS have linked approximately 60 genetic loci to $\mathrm{CAD} .{ }^{41}$ Although coagulation plays a crucial role in CAD pathophysiology, very few of these GWASidentified loci have been related with haemostatic processes so far. ${ }^{42,43}$ The here reported association suggests that one of the SNPs with the strongest association with CAD identified by GWAS, ${ }^{25}$ beyond the effects on lipid metabolism, may influence also blood coagulation.

Our results, supporting a connection between ApoC-III/ TRLs and pro-thrombotic diathesis marked by FVIIa-AT, may have implications for both arterial and venous thrombosis. As a matter of fact, high levels of FVIIa-AT have been observed in patients with venous thromboembolism (VTE), ${ }^{15}$ and hypertriglyceridaemia has been also related with $\mathrm{VTE}^{44}$ and lipid-lowering therapies may reduce VTE risk. ${ }^{45}$

It is important to underline some limitations of this work. First, our results may be intrinsically flawed by the crosssectional design. Statistical association does not mean biological causality and no insights on the possible causal mechanisms linking ApoC-III and coagulation can be inferred. It could be argued that apolipoproteins could remodel the phospholipid distribution in cellular membranes, ${ }^{46,47}$ which in turn influences TF-FVIIa activity at the cell surface. ${ }^{48-50}$ However, our work does not provide any proof about the molecular mechanisms potentially linking ApoC-III and TF-FVIIa interaction. It could be not excluded that ApoC-III plasma levels may merely alter either directly or indirectly the clearance of FVIIa-AT complex (e.g. by interfering with hepatocyte-related clearance), without any significant effects on TF-FVIIa interaction. Nonetheless, the present result, consistent with previous works ${ }^{22,23}$ demonstrating that high ApoC-III concentrations are associated with an increased activation of extrinsic and common pathways of coagulation cascade (-Supplementary Fig. S3, available in the online version), suggests a link between ApoC-III and pro-coagulant diathesis more than an influence on clearance of the inactive FVIIa-AT complex. As further limitations, the population sample was limited and the polymorphisms for genetic analysis were selected on the basis of a priori hypothesis (i.e. gene variants known to influence the levels of apolipoproteins and plasma lipids). It should be noted that a recent GWAS identifies only F7 promoter region and PROCR gene as loci associated with circulating levels of FVIIa-AT. ${ }^{18}$ Finally, we recognize the lack of some laboratory parameters, like other apolipoproteins which are known to be involved in TRLs metabolism (e.g. ApoC-II and ApoA-V).

In summary, our results indicate a strong association between ApoC-III and FVIIa-AT, thereby suggesting that an increased ApoC-III concentration may identify subjects with a pro-thrombotic diathesis characterized by an enhanced TFFVIIa interaction. Our results should be confirmed by further studies, both investigating the underlying molecular mechanisms and validating our findings in larger populations. Nonetheless, they support the fascinating hypothesis of an additional and clinically relevant link between plasma lipids and coagulation which may pave the way to new approaches in the management of CVD.

\section{What is known about this topic?}

- Plasma lipoproteins can stimulate coagulation cascade, with triglyceride-rich lipoproteins (TRLs) being apparently the most efficient.

- Tissue factor (TF) and activated factor VII (FVIIa) are involved in phospholipid interactions which enhance their coagulant activity.

- Activated factor VII-anti-thrombin complex (FVIIa-AT) in plasma may reflect tissue factor (TF) exposure and TF-FVIIa interaction.

\section{What does this paper add?}

- Apolipoprotein C-III (ApoC-III), a crucial player of TRLs metabolism, showed the strongest correlation with FVIIa-AT among all plasma lipid parameters.

- The APOC3-tagging polymorphism rs964184, linked by GWAS with cardiovascular diseases, was consistently associated with both ApoC-III and FVIIa-AT plasma levels.

- An increased ApoC-III concentration may identify subjects with a pro-thrombotic diathesis characterized by an enhanced TF-FVIIa interaction.

\section{Funding}

This work was supported by the Cariverona Foundation (project B36J16002570003), Verona, Italy, and by the grants 2010XE5L2R_002 (MIUR) and 1786/2012 Strategic Research Program of Emilia Romagna Region, Italy.

Conflict of Interest

$\mathrm{N}$. Martinelli reports having received a payment from Diagnostica Stago, Asnieres, France, for management and conduct of the study. B. Woodhams reports having been a consultant for Diagnostica Stago via Haemacon Ltd. The other authors state that they have no conflict of interest.

\section{Acknowledgements}

We are grateful to Diagnostica Stago, Asnieres, France, for financial support and for providing the reagents for the FVIIa-AT assay.

We thank Prof. Sekar Kathiresan (Co-director, Program in Medical \& Population Genetics, Broad Institute; Associate Professor of Medicine, Harvard Medical School; Director, Center for Genomic Medicine, Massachusetts General Hospital, Boston, MA) for the kind cooperation and for providing the data about rs964184 genotype. We thank 
Maria Zoppi for her invaluable secretarial help and Diego Minguzzi and Patrizia Pattini for their excellent technical help. This study was performed in part in the LURM (Laboratorio Universitario di Ricerca Medica) Research Center, University of Verona.

\section{References}

1 Libby P, Ridker PM, Hansson GK. Progress and challenges in translating the biology of atherosclerosis. Nature 2011;473 (7347):317-325

2 Reiner Ž. Statins in the primary prevention of cardiovascular disease. Nat Rev Cardiol 2013;10(08):453-464

3 Reiner Ž. Hypertriglyceridaemia and risk of coronary artery disease. Nat Rev Cardiol 2017;14(07):401-411

4 Muller MP, Wang Y, Morrissey JH, Tajkhorshid E. Lipid specificity of the membrane binding domain of coagulation factor X. J Thromb Haemost 2017;15(10):2005-2016

$5 \mathrm{Ke} \mathrm{K}$, Yuan J, Morrissey JH. Tissue factor residues that putatively interact with membrane phospholipids. PLoS One 2014;9(02): e88675

6 Hemker HC, Esnouf MP, Hemker PW, Swart AC, Macfarlane RG. Formation of prothrombin converting activity. Nature 1967;215 (5098):248-251

7 Hoffman CJ, Lawson WE, Miller RH, Hultin MB. Correlation of vitamin K-dependent clotting factors with cholesterol and triglycerides in healthy young adults. Arterioscler Thromb 1994;14 (11): $1737-1740$

8 Tosi F, Micaglio R, Sandri M, et al. Increased plasma thrombin potential is associated with stable coronary artery disease: an angiographically-controlled study. Thromb Res 2017;155:16-22

9 Rota S, McWilliam NA, Baglin TP, Byrne CD. Atherogenic lipoproteins support assembly of the prothrombinase complex and thrombin generation: modulation by oxidation and vitamin $\mathrm{E}$. Blood 1998;91(02):508-515

10 Moyer MP, Tracy RP, Tracy PB, van't Veer C, Sparks CE, Mann KG. Plasma lipoproteins support prothrombinase and other procoagulant enzymatic complexes. Arterioscler Thromb Vasc Biol 1998; 18(03):458-465

11 Morrissey JH. Tissue factor: a key molecule in hemostatic and nonhemostatic systems. Int J Hematol 2004;79(02):103-108

12 Chen VM, Hogg PJ. Encryption and decryption of tissue factor. J Thromb Haemost 2013;11(Suppl 1):277-284

13 Chiu J, Passam F, Butera D, Hogg PJ. Protein disulfide isomerase in thrombosis. Semin Thromb Hemost 2015;41(07):765-773

14 Morissey JH. Assay for Measuring FVIIa-Antithrombin Complexes. Oklahoma City, OK: Oklahoma Medical Research Foundation; 2003

15 Spiezia L, Rossetto V, Campello E, et al. Factor VIla-antithrombin complexes in patients with arterial and venous thrombosis. Thromb Haemost 2010;103(06):1188-1192

16 Silveira A, Scanavini D, Boquist S, et al. Relationships of plasma factor VIIa-antithrombin complexes to manifest and future cardiovascular disease. Thromb Res 2012;130(02):221-225

17 Martinelli N, Girelli D, Baroni M, et al. Activated factor VIIantithrombin complex predicts mortality in patients with stable coronary artery disease: a cohort study. J Thromb Haemost 2016; 14(04):655-666

18 Olson NC, Raffield LM, Lange LA, et al. Associations of activated coagulation factor VII and factor VIIa-antithrombin levels with genome-wide polymorphisms and cardiovascular disease risk. J Thromb Haemost 2018;16(01):19-30

19 Martinelli N, Girelli D, Lunghi B, et al. Polymorphisms at LDLR locus may be associated with coronary artery disease through modulation of coagulation factor VIII activity and independently from lipid profile. Blood 2010;116(25):5688-5697
20 Levey AS, Bosch JP, Lewis JB, Greene T, Rogers N, Roth D; Modification of Diet in Renal Disease Study Group. A more accurate method to estimate glomerular filtration rate from serum creatinine: a new prediction equation. Ann Intern Med 1999;130(06):461-470

21 Martinelli N, Olivieri O, Shen GQ et al. Additive effect of LRP8/ APOER2 R952Q variant to APOE epsilon2/epsilon3/epsilon4 genotype in modulating apolipoprotein $\mathrm{E}$ concentration and the risk of myocardial infarction: a case-control study. BMC Med Genet 2009;10:41

22 Olivieri O, Martinelli N, Girelli D, et al. Apolipoprotein C-III predicts cardiovascular mortality in severe coronary artery disease and is associated with an enhanced plasma thrombin generation. J Thromb Haemost 2010;8(03):463-471

23 Olivieri O, Martinelli N, Baroni M, et al. Factor II activity is similarly increased in patients with elevated apolipoprotein CIII and in carriers of the factor II 20210A allele. J Am Heart Assoc 2013;2(06): e000440

24 Kathiresan S, Willer CJ, Peloso GM, et al. Common variants at 30 loci contribute to polygenic dyslipidemia. Nat Genet 2009;41(01): 56-65

25 Schunkert H, König IR, Kathiresan S, et al; Cardiogenics; CARDIoGRAM Consortium. Large-scale association analysis identifies 13 new susceptibility loci for coronary artery disease. Nat Genet 2011;43(04):333-338

26 Webb TR, Erdmann J, Stirrups KE, et al; Wellcome Trust Case Control Consortium; MORGAM Investigators; Myocardial Infarction Genetics and CARDIoGRAM Exome Consortia Investigators. Systematic evaluation of pleiotropy identifies 6 further loci associated with coronary artery disease. J Am Coll Cardiol 2017;69(07):823-836

27 Greenow K, Pearce NJ, Ramji DP. The key role of apolipoprotein E in atherosclerosis. J Mol Med (Berl) 2005;83(05):329-342

28 Fallaize R, Carvalho-Wells AL, Tierney AC, et al. APOE genotype influences insulin resistance, apolipoprotein CII and CIII according to plasma fatty acid profile in the metabolic syndrome. Sci Rep 2017;7(01):6274

29 Olivieri O, Martinelli N, Bassi A, et al. ApoE epsilon2/epsilon3/ epsilon4 polymorphism, ApoC-III/ApoE ratio and metabolic syndrome. Clin Exp Med 2007;7(04):164-172

30 Wyler von Ballmoos MC, Haring B, Sacks FM. The risk of cardiovascular events with increased apolipoprotein CIII: a systematic review and meta-analysis. J Clin Lipidol 2015;9(04):498-510

31 Pechlaner R, Tsimikas S, Yin X, et al. Very-low-density lipoproteinassociated apolipoproteins predict cardiovascular events and are lowered by inhibition of APOC-III. J Am Coll Cardiol 2017;69(07): 789-800

32 Sacks FM. The crucial roles of apolipoproteins E and C-III in apoB lipoprotein metabolism in normolipidemia and hypertriglyceridemia. Curr Opin Lipidol 2015;26(01):56-63

33 Crosby J, Peloso GM, Auer PL, et al; TG and HDL Working Group of the Exome Sequencing Project, National Heart, Lung, and Blood Institute. Loss-of-function mutations in APOC3, triglycerides, and coronary disease. N Engl J Med 2014;371(01):22-31

34 Gaudet D, Alexander VJ, Baker BF, et al. Antisense inhibition of apolipoprotein C-III in patients with hypertriglyceridemia. N Engl J Med 2015;373(05):438-447

35 Jensen MK, Aroner SA, Mukamal KJ, et al. High-density lipoprotein subspecies defined by presence of apolipoprotein C-III and incident coronary heart disease in four cohorts. Circulation 2018;137 (13):1364-1373

36 Morton AM, Koch M, Mendivil CO, et al. Apolipoproteins E and CIII interact to regulate HDL metabolism and coronary heart disease risk. JCI Insight 2018;3(04):98045

37 Kjalke M, Silveira A, Hamsten A, Hedner U, Ezban M. Plasma lipoproteins enhance tissue factor-independent factor VII activation. Arterioscler Thromb Vasc Biol 2000;20(07):1835-1841 
38 Silveira A, Carlo A, Adam M, et al. VIIaAT complexes, procoagulant phospholipids, and thrombin generation during postprandial lipemia. Int J Lab Hematol 2018;40(03):251-257

39 Archer WR, Desroches S, Lamarche B, et al. Variations in plasma apolipoprotein C-III levels are strong correlates of the triglyceride response to a high-monounsaturated fatty acid diet and a highcarbohydrate diet. Metabolism 2005;54(10):1390-1397

40 Parra EJ, Mazurek A, Gignoux CR, et al. Admixture mapping in two Mexican samples identifies significant associations of locus ancestry with triglyceride levels in the BUD13/ZNF259/APOA5 region and fine mapping points to rs964184 as the main driver of the association signal. PLoS One 2017;12(02):e0172880

41 Khera AV, Kathiresan S. Genetics of coronary artery disease: discovery, biology and clinical translation. Nat Rev Genet 2017; 18(06):331-344

42 Reilly MP, Li M, He J, et al; Myocardial Infarction Genetics Consortium; Wellcome Trust Case Control Consortium. Identification of ADAMTS7 as a novel locus for coronary atherosclerosis and association of $\mathrm{ABO}$ with myocardial infarction in the presence of coronary atherosclerosis: two genome-wide association studies. Lancet 2011;377(9763):383-392

43 Howson JMM, Zhao W, Barnes DR, et al; CARDIoGRAMplusC4D; EPIC-CVD. Fifteen new risk loci for coronary artery disease highlight arterial-wall-specific mechanisms. Nat Genet 2017;49(07): 1113-1119
44 Ageno W, Becattini C, Brighton T, Selby R, Kamphuisen PW. Cardiovascular risk factors and venous thromboembolism: a meta-analysis. Circulation 2008;117(01):93-102

45 Kunutsor SK, Seidu S, Khunti K. Statins and primary prevention of venous thromboembolism: a systematic review and meta-analysis. Lancet Haematol 2017;4(02):e83-e93

46 Cardin AD, Jackson RL, Johnson JD. Effects of human plasma apolipoproteins C-I, C-II and C-III on the phase-transition of sonicated vesicles of dipalmitoylphosphatidylcholine. FEBS Lett 1982;141(02):193-197

47 Hanson CL, Ilag LL, Malo J, Hatters DM, Howlett GJ, Robinson CV. Phospholipid complexation and association with apolipoprotein C-II: insights from mass spectrometry. Biophys J 2003;85(06): 3802-3812

48 Hathcock JJ, Rusinova E, Gentry RD, Andree H, Nemerson Y. Phospholipid regulates the activation of factor $X$ by tissue factor/factor VIIa (TF/VIIa) via substrate and product interactions. Biochemistry 2005;44(22):8187-8197

49 Mariani G, Conard J, Bernardi F, et al. Oral contraceptives highlight the genotype-specific association between serum phospholipids and activated factor VII. Arterioscler Thromb Vasc Biol 1999;19 (08):2024-2028

50 Morrissey JH, Davis-Harrison RL, Tavoosi N, et al. Protein-phospholipid interactions in blood clotting. Thromb Res 2010;125 (Suppl 1):S23-S25 